УДК 517.9

\title{
A Sufficient Condition for Absolute Continuity of Conjugations between Interval Exchange Maps
}

\author{
Abdumajid S. Begmatov* \\ National University of Uzbekistan \\ University street 4, Olmazor district, Tashkent, 100174 \\ Turin Polytechnic University in Tashkent \\ Niyazov Str. 17, Tashkent, 100095 \\ Uzbekistan
}

Received 29.12.2018, received in revised form 11.01.2019, accepted 06.02.2019

A class of topological equivalent generalized interval exchange maps of genus one and of the same bounded combinatorics is considered in the paper. A sufficient condition for absolute continuity of the conjugation between two maps from this class is provided.

Keywords: conjugate map, interval exchange map, Rauzy-Veech induction, renormalization, dynamical partition, martingale.

DOI: $10.17516 / 1997-1397-2019-12-3-276-284$.

\section{Introduction and preliminaries}

The study of ergodic properties of interval exchange maps (i.e.m.) is a classical problem of dynamical systems. In recent years interest in this topic has been renewed (see [1-6]).

A standard i.e.m. $f$ on an finite length interval $I$ is a one-to-one map which is locally translation except at a finite number of discontinuities. The generalized i.e.m. obtained by replacing linear mappings of subintervals with a locally orientation-preserving homeomorphism. Let $d$ be the number of intervals of continuity of $f$. When $d=2$ standard i.e.m. correspond to linear rotations of the circle, and generalized i.e.m. correspond to homeomorphisms of the circle with two break points. Generalized interval exchange maps were introduced [5]. It was showed that sufficiently smooth generalized i.e.m. of a certain combinatorial type (deformations of standard interval exchange transformations and tangent to them at the points of discontinuities) are smoothly linearizable. Considering piecewise $C^{2+\varepsilon}$-smooth circle homeomorphisms as generalized interval exchange maps of genus one, Cunha and Smania [3] showed that two generalized i.e.m. with the same bounded-type combinatorics and zero mean nonlinearities $C^{1}$-smoothly conjugate to each other. In the case of circle maps it was shown that for almost all rotation numbers every two $C^{2+\varepsilon}$-smooth circle homeomorphisms with a break point, with the same irrational rotation number and the same size of the break are $C^{1}$-smoothly conjugate to each other [7-9]. Let us note that statement on the regularity of conjugating map can be obtained by using the convergence of renormalizations of given maps.

The lower bound of the scale of smoothness for a homeomorphism $f$, that is, $D f$ is absolutely continuous and $D \log D f \in \mathbb{L}_{p}$ for some $p>1$ was considered [10,11]. The latter conditions on smoothness of $f$ are called the Katznelson and Ornstein (KO, for short) smoothness condition [12]. For this low smoothness case in it was shown that the Rauzy-Veech renormalizations of two piecewise KO-smooth maps satisfying certain combinatorial assumptions approach to each other in $C^{1+L_{1}}$-norm [11]. Let us note that the $\mathrm{KO}$ smoothness condition is smaller than $C^{2+\nu}$ smoothness but the obtained convergence rate is slower than exponential $[3,7]$. However, in

*abdumajidb@gmail.com

(c) Siberian Federal University. All rights reserved 
this case one can expect the absolutely continuity (not $C^{1}$ rigidity) of conjugate map between two generalized i.e.m.. Our objective is to obtain a sufficient condition for conjugate map to be absolutely continuous.

Let us define a class of generalized interval exchange maps. Let $\mathbb{B}^{K O}$ be the set of g.i.e.m. $f: I \rightarrow I$ such that

(i) the map $f$ has cyclic permutation;

(ii) the map $f$ has no connection and has $k$ - bounded combinatorics.

(iii) on each intervals of continuity of $f$ the map $f$ satisfies the Katznelson and Ornstein smoothness condition: $D f$ is absolutely continuous and $D \ln D f \in L_{p}$ for some $p>1$.

Conditions $(i)$ and $(i i)$ are explained in Section 2. Let us note that the class $\mathbb{B}^{K O}$ consists of circle homeomorphisms with several break points and with irrational rotation number of bounded type.

Two g.i.e.m. $f_{1}, f_{2} \in \mathbb{B}^{K O}$ with the same combinatorics are called break-equivalent if the following conditions hold true:

(a) the break points of one map $u_{i}$ are mapped into the break points of the other map $v_{i}$ by a topological conjugacy $h$ satisfying $f_{2}=h^{-1} \circ f_{1} \circ h$, i.e., $u_{i}=h\left(v_{i}\right)$;

(b) the corresponding sizes of breaks $c_{i}=\sqrt{f_{1}^{\prime}\left(u_{i}-0\right) / f_{1}^{\prime}\left(u_{i}+0\right)}, \widetilde{c}_{i}=\sqrt{f_{2}^{\prime}\left(v_{i}-0\right) / f_{2}^{\prime}\left(v_{i}+0\right)}$ are the same for each $i=1, \ldots, k$.

Let $f_{1}$ and $f_{2}$ be break-equivalent g.i.e.m. of class $\mathbb{B}^{K O}$. Let us consider dynamical partitions $\xi_{n}\left(f_{1}\right)$ and $\xi_{n}\left(f_{2}\right)$ generated by maps $f_{1}$ and $f_{2}$. Let $h$ be a conjugation homeomorphism between $f_{1}$ and $f_{2}$, that is, $h \circ f_{1}=f_{2} \circ h$. Let us assume that $\Delta^{(n)}$ are elements of the partition of $\xi_{n}\left(f_{2}\right)$. Since $h$ is a conjugate map between $f_{1}$ and $f_{2}$ for any $L^{(n)} \in \xi_{n}\left(f_{1}\right)$, we have $h\left(L^{(n)}\right)=\Delta^{(n)}$ and $\Delta^{(n)} \in \xi_{n}\left(f_{2}\right)$. The main result is the following

Theorem 1.1. Let $f_{1}$ and $f_{2}$ be break-equivalent g.i.e.m. of class $\mathbb{B}^{K O}$. Suppose that there exist a sequence $\delta_{n}$ with $\sum_{n=1}^{\infty} \delta_{n}^{2}<\infty$ such that

$$
\left|\frac{\left|h\left(L^{(n)}\right)\right|}{\left|h\left(R^{(n)}\right)\right|}-\frac{\left|L^{(n)}\right|}{\left|R^{(n)}\right|}\right| \leqslant \delta_{n},
$$

for each pair of adjacent intervals $L^{(n)}, R^{(n)} \in \xi_{n}\left(f_{1}\right)$. Then the conjugate map $h$ is absolutely continuous function.

The structure of the paper is as follows. In Section 2 we present necessary definitions on interval exchange maps and define a renormalization map related to Rauzy-Veech induction. In Section 3 we define a sequence of dynamical partition associated with renormalization map and formulate statements on asymptotic lengths of the elements of dynamical partition. Finally, in Section 4 we define a martingale, and using its properties, prove our main theorem.

\section{Background on the interval exchange maps}

In this section we describe combinatorial assumptions on the class $\mathbb{B}^{K O}$. Let $I$ be an open bounded interval and $\mathcal{A}$ be an alphabet with $d \geqslant 2$ symbols. Let us consider the partition of $I$ into $d$ subintervals indexed by $\mathcal{A}$, that is, $\mathcal{P}=\left\{I_{\alpha}, \alpha \in \mathcal{A}\right\}$. Let $f: I \rightarrow I$ be a bijection. We say that the triple $(f, \mathcal{A}, \mathcal{P})$ is a generalized interval exchange map with $d$ intervals (for short g.i.e.m.) if $\left.f\right|_{I_{\alpha}}$ is an orientation-preserving homeomorphism for all $\alpha \in \mathcal{A}$. If $\left.f\right|_{I_{\alpha}}$ is a translation then $f$ is called a standard interval exchange map (for short s.i.e.m.). 
Let $f: I \rightarrow I$ be a g.i.e.m. with alphabet $\mathcal{A}$ and $\pi_{0}, \pi_{1}: \mathcal{A} \rightarrow\{1, \ldots, d\}$, be bijections such that $\pi_{0}(\alpha)<\pi_{0}(\beta)$ iff $I_{\alpha}<I_{\beta}$ and $\pi_{1}(\alpha)<\pi_{1}(\beta)$ if $f\left(I_{\alpha}\right)<f\left(I_{\beta}\right)$.

Pair $\pi=\left(\pi_{0}, \pi_{1}\right)$ is called the combinatorial data associated with g.i.e.m. $f$. The notation $\pi=$ $(\pi(1), \pi(2), \ldots, \pi(d))$ is also used for the combinatorial data of $f$. It is always assumed that pair $\pi=\left(\pi_{0}, \pi_{1}\right)$ is irreducible, that is, for all $j \in\{1, \ldots, d-1\}$ we have $\pi_{0}^{-1}(1, \ldots, j) \neq \pi_{1}^{-1}(1, \ldots, j)$. It is assumed that g.i.e.m. $f$ has cyclic permutation if $\pi_{0}(\{1,2, \ldots, d\})=\{j+1, \ldots, d, 1, \ldots, j\}$ for some $1 \leqslant j \leqslant d-1$.

Let $\pi=\left(\pi_{0}, \pi_{1}\right)$ be the combinatorial data associated with g.i.e.m $f$. For each $\varepsilon \in\{0,1\}$ the last symbol in the expression of $\pi_{\varepsilon}$ is denoted by $\alpha(\varepsilon)=\pi_{\varepsilon}^{-1}(d)$.

Let us assume that intervals $I_{\alpha(0)}$ and $f\left(I_{\alpha(1)}\right)$ have different lengths. Then g.i.e.m. $f$ is called Rauzy-Veech renormalizable(renormalizable, for short). If $\left|I_{\alpha(0)}\right|>\left|f\left(I_{\alpha(1)}\right)\right|$ then $f$ is renormalizable of type 0 . When $\left|I_{\alpha(0)}\right|<\left|f\left(I_{\alpha(1)}\right)\right|$ then $f$ is renormalizable of type 1 . In either case, the letter corresponding to the largest of these intervals is called winner and the letter corresponding to the shortest of these intervals is called loser of $\pi$. Let $I^{(1)}$ be the subinterval of $I$ obtained by removing the loser, that is, the shortest of these two intervals:

$$
I^{(1)}= \begin{cases}I \backslash f\left(I_{\alpha(1)}\right), & \text { if type } 0, \\ I \backslash I_{\alpha(0)}, & \text { if type } 1 .\end{cases}
$$

Since the loser is the last subinterval on the right of $I$, the intervals $I$ and $I^{(1)}$ have the same left endpoint.

The Rauzy-Veech induction of $f$ is the first return map $R(f)$ to the subinterval $I^{(1)}$. Let us assume that $R(f)$ is again g.i.e.m. with the same alphabet $\mathcal{A}$. For this one need to associate with this map an $\mathcal{A}$ - indexed partition of its domain. Subintervals of $I^{(1)}$ are denoted by $I_{\alpha}^{(1)}$. Let $f$ be renormalizable of type 0 . Then interval $I^{(1)}=I \backslash f\left(I_{\alpha(1)}\right)$ is the domain of $R(f)$ and we have

$$
I_{\alpha}^{(1)}=\left\{\begin{array}{l}
I_{\alpha}, \quad \text { for } \alpha \neq \alpha(0), \\
I_{\alpha(0)} \backslash f\left(I_{\alpha(1)}\right), \quad \text { for } \alpha=\alpha(0) .
\end{array}\right.
$$

These intervals form a partition of the interval $I^{(1)}$ and they are denoted by $\mathcal{P}^{(1)}=\left\{I_{\alpha}^{(1)}, \alpha \in \mathcal{A}\right\}$. Since $f\left(I_{\alpha(1)}\right)$ is the last interval on the right of $f(\mathcal{P})$ we have $f\left(I_{\alpha}^{(1)}\right) \subset I^{(1)}$ for every $\alpha \neq \alpha(1)$. This means that $R(f):=f$ is restricted to these $I_{\alpha}^{(1)}$. On the other hand, because $I_{\alpha(1)}^{(1)}=I_{\alpha(1)}$ we have

$$
f\left(I_{\alpha(1)}^{(1)}\right)=f\left(I_{\alpha(1)}\right) \subset I_{\alpha(0)}, \quad \text { and so } f^{2}\left(I_{\alpha(1)}^{(1)}\right) \subset f\left(I_{\alpha(0)}\right) \subset I^{(1)} .
$$

Then $R(f):=f^{2}$ is restricted to $I_{\alpha(1)}^{(1)}$. Thus

$$
R(f)(x)=\left\{\begin{array}{ll}
f(x), & \text { if } \quad x \in I_{\alpha}^{(1)} \\
f^{2}(x), & \text { if } \quad x \in I_{\alpha(1)}^{(1)}
\end{array} \text { and } \alpha \neq \alpha(1)\right.
$$

If $f$ is renormalizable of type 1 then interval $I^{(1)}=I \backslash I_{\alpha(0)}$ is the domain of $R(f)$, we have



Then $f\left(I_{\alpha}^{(1)}\right) \subset I^{(1)}$ for every $\alpha \neq \alpha(0)$, and so $R(f)=f$ is restricted to these $I_{\alpha}^{(1)}$. On the other hand,

$$
f^{2}\left(I_{\alpha(0)}^{(1)}\right)=f\left(I_{\alpha(0)}\right) \subset I^{(1)}
$$


and $R(f)=f^{2}$ is restricted to $I_{\alpha(0)}^{(1)}$. Thus,

$$
R(f)(x)=\left\{\begin{array}{ll}
f(x), & \text { if } x \in I_{\alpha}^{(1)} \\
f^{2}(x), & \text { if } x \in I_{\alpha(0)}^{(1)}
\end{array} \text { and } \alpha \neq \alpha(0)\right.
$$

It is easy to see that $R(f)$ is a bijection on $I^{(1)}$ and an orientation-preserving homeomorphisms on each $I_{\alpha}^{(1)}$. Moreover, the alphabet $\mathcal{A}$ for $f$ and $R(f)$ is the same.

The triple $\left(R(f), \mathcal{A}, \mathcal{P}^{1}\right)$ is called the Rauzy-Veech renormalization of $f$. If $f$ is renormalizable of type $\varepsilon \in\{0,1\}$ then combinatorial data $\pi^{1}=\left(\pi_{0}^{1}, \pi_{1}^{1}\right)$ of $R(f)$ are given by

$$
\pi_{\varepsilon}^{1}:=\pi_{\varepsilon}, \quad \text { and } \quad \pi_{1-\varepsilon}^{1}(\alpha)= \begin{cases}\pi_{1-\varepsilon}(\alpha), & \text { if } \pi_{1-\varepsilon}(\alpha) \leqslant \pi_{1-\varepsilon}(\alpha(\varepsilon)) \\ \pi_{1-\varepsilon}(\alpha)+1, & \text { if } \pi_{1-\varepsilon}(\alpha(\varepsilon))<\pi_{1-\varepsilon}(\alpha)<d \\ \pi_{1-\varepsilon}(\alpha(\varepsilon))+1, & \text { if } \pi_{1-\varepsilon}(\alpha)=d\end{cases}
$$

We say that g.i.e.m. $f$ is infinitely renormalizable if $R^{n}(f)$ is well defined for every $n \in \mathbb{N}$. Let $I^{(n)}$ be the domain of $R^{n}(f)$. It is clear that $R^{n}(f)$ is the first return map for $f$ to the interval $I^{(n)}$. Similarly, $R^{n}(f)^{-1}=R^{n}\left(f^{-1}\right)$ is the first return map for $f$ to the interval $I^{(n)}$.

For every interval of the form $J=[a, b)$ we put $\partial J:=\{a\}$.

Definition 1. We say that g.i.e.m. f has no connection if

$$
f^{m}\left(\partial I_{\alpha}\right) \neq \partial I_{\beta}, \quad \text { for all } m \geqslant 1 \quad \text { and } \quad \alpha, \beta \in \mathcal{A} \quad \text { with } \quad \pi_{0}(\beta) \neq 1 \text {. }
$$

It is clear that $f\left(\partial I_{\alpha}\right)=\partial I_{\beta}$ for $\alpha=\pi_{1}^{-1}(1)$ in the case $\pi_{0}(\beta)=1$. Condition (6) is called the Keane condition. Keane [13] showed that no connection condition is a necessary and sufficient condition for $f$ to be infinitely renormalizable. Condition (6) means that the orbits of the left end point of subintervals $I_{\alpha}, \alpha \in \mathcal{A}$ are disjoint whenever they can be.

Let $\varepsilon_{n}$ be the type of the $n$-th renormalization and let $\alpha_{n}\left(\varepsilon_{n}\right)$ be the winner and $\alpha_{n}\left(1-\varepsilon_{n}\right)$ be the loser of the $n$-th renormalization.

Definition 2. We say that g.i.e.m. $f$ has $k$-bounded combinatorics if for each $n \in \mathbb{N}$ and $\beta, \gamma \in \mathcal{A}$ there exist $n_{1}, p \geqslant 0$ with $\left|n-n_{1}\right|<k$ and $\left|n-n_{1}-p\right|<k$ such that

$$
\begin{gathered}
\alpha_{n_{1}}\left(\varepsilon_{n_{1}}\right)=\beta, \alpha_{n_{1}+p}\left(1-\varepsilon_{n_{1}+p}\right)=\gamma, \text { and } \\
\alpha_{n_{1}+i}\left(1-\varepsilon_{n_{1}+p}\right)=\alpha_{n_{1}+i+1}\left(\varepsilon_{n_{1}+i}\right), \quad \text { for every } 0 \leqslant i<p .
\end{gathered}
$$

We say that g.i.e.m. $f: I \rightarrow I$ has genus one (or belongs to the rotation class) if $f$ has at most two discontinuities. Let us note that every g.i.e.m. with either two or three intervals has genus one, and the genus of g.i.e.m. is invariant under renormalization.

\section{Dynamical partitions associated with interval exchange map}

Let $(f, \mathcal{A}, \mathcal{P})$ be a g.i.e.m. with $d$ intervals, and $\mathcal{P}=\left\{I_{\alpha}: \alpha \in \mathcal{A}\right\}$ be the initial $\mathcal{A}$ indexed partition of $I$. Interval $I=[0,1)$ is taken for definiteness. Suppose that $f$ is infinitely renormalizable. Let $I^{(n)}$ be the domain of $R^{n}(f)$. Let us note that $I^{(n)}$ is the nested sequence of subintervals, and it has the same left endpoint of $I$. We want to construct the dynamical partition of $I$ associated with the domain of $R^{n}(f)$.

As mentioned above, $R(f)$ is g.i.e.m. with $d$ intervals and intervals $I_{\alpha}^{(1)}$ generate an $\mathcal{A}$ indexed partition of $I^{(1)}$ denoted by $\mathcal{P}^{1}$. One can verify by induction that $R^{n}(f)$ is g.i.e.m. with 
$d$ intervals. Let $\mathcal{P}^{n}=\left\{I_{\alpha}^{(n)}: \alpha \in \mathcal{A}\right\}$ be the $\mathcal{A}$ - indexed partition of $I^{(n)}$ generated by $R^{n}(f)$. $\mathcal{P}^{n}$ is called the fundamental partition and $I_{\alpha}^{(n)}$ is called the fundamental segments of rank $n$.

Since $R^{n}(f)$ is the first return map for $f$ to the interval $I^{(n)}$, each fundamental segment $I_{\alpha}^{(n)} \in \mathcal{P}^{n}$ returns to $I^{(n)}$ under certain iterates of the map $f$. Prior to returning, these intervals are in the interval $I \backslash I^{(n)}$ for some time. Consequently the system of intervals (their interiors are mutually disjoint)

$$
\xi_{n}=\left\{f^{i}\left(I_{\alpha}^{(n)}\right), 0 \leqslant i \leqslant q_{\alpha}^{n}-1, \alpha \in \mathcal{A}\right\}
$$

covers the whole interval and forms a partition of $I$.

The system of intervals $\xi_{n}$ is called the $n$-th dynamical partition of $I$. The dynamical partitions $\xi_{n}$ are refined with increasing $n$, where $\xi_{n+1} \supset \xi_{n}$ means that any element of the preceding partition is a union of a number of elements of the next partition or belongs to the next partition. Let us denote by $\xi_{n+1}^{p r}$ the system of preserved intervals of $\xi_{n}$. More precisely, if $R^{n} f$ has type 0

$$
\xi_{n+1}^{p r}=\left\{f^{i}\left(I_{\alpha}^{(n)}\right), 0 \leqslant i \leqslant q_{\alpha}^{n}-1, \text { for } \alpha \neq \alpha(0)\right\},
$$

and if $R^{n} f$ has type 1

$$
\xi_{n+1}^{p r}=\left\{f^{i}\left(I_{\alpha}^{(n)}\right), 0 \leqslant i \leqslant q_{\alpha}^{n}-1, \text { for } \alpha \neq \alpha(1)\right\} .
$$

Let $\xi_{n+1}^{t n}:=\xi_{n+1} \backslash \xi_{n+1}^{p r}$ be the set of elements of $\xi_{n+1}$ which are properly contained in some element of $\xi_{n}$. Therefore, if $R^{n} f$ has type 0

$$
\begin{aligned}
& \xi_{n+1}^{t n}=\left\{f^{i}\left(I_{\alpha(0)}^{(n+1)}\right), 0 \leqslant i<q_{\alpha(0)}^{n}\right\} \bigcup\left\{f^{i}\left(I_{\alpha(1)}^{(n+1)}\right), 0 \leqslant i<q_{\alpha(0)}^{n}\right\}= \\
& =\bigcup_{i=0}^{q_{\alpha(0)}^{n}-1}\left\{f^{i}\left(I_{\alpha(0)}^{(n)} \backslash f^{q_{\alpha(1)}^{n}} I_{\alpha(1)}^{(n)}\right)\right\} \bigcup \bigcup_{i=q_{\alpha(1)}^{n}}^{q_{\alpha(1)}^{n}+q_{\alpha(0)}^{n}-1}\left\{f^{i}\left(I_{\alpha(1)}^{(n)}\right)\right\},
\end{aligned}
$$

and if $R^{n} f$ has type 1

$$
\begin{aligned}
& \xi_{n+1}^{t n}=\left\{f^{i}\left(I_{\alpha(0)}^{(n+1)}\right), 0 \leqslant i<q_{\alpha(1)}^{n}\right\} \bigcup\left\{f^{i}\left(I_{\alpha(1)}^{(n+1)}\right), 0 \leqslant i<q_{\alpha(1)}^{n}\right\}=
\end{aligned}
$$

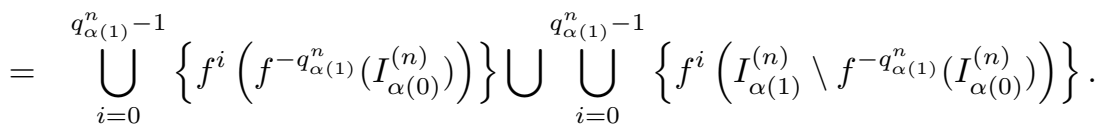

So, the partition $\xi_{n+1}$ consists of preserving elements of $\xi_{n}$ and images of two (new) intervals for defining $R^{n+1}(f)$, that is, $\xi_{n+1}=\xi_{n+1}^{p r} \cup \xi_{n+1}^{t n}$. Let us also note that for the first return time $q_{\alpha}^{n}$ we have:

(1) if $\alpha=\alpha^{n}(\varepsilon)$, then $q_{\alpha^{n}(\varepsilon)}^{n+1}=q_{\alpha^{n}(\varepsilon)}^{n}$;

(2) if $\alpha=\alpha^{n}(1-\varepsilon)$, then $q_{\alpha^{n}(1-\varepsilon)}^{n+1}=q_{\alpha^{n}(1-\varepsilon)}^{n}+q_{\alpha^{n}(\varepsilon)}^{n}$.

Bounded geometry. Let us denote the set of g.i.e.m $f: I \rightarrow I$ by $\mathbb{B}^{1+b v}$. It satisfies conditions (i) - (ii) which are piecewise $C^{1}$ - smooth and have bounded variation of the first derivative.

The norm of the dynamical partition $\xi_{n}$ is $\left\|\xi_{n}\right\|=\max \left\{\left|f^{i}\left(I_{\alpha}^{n}\right)\right|\right\}$, where the maximum is taken over all $\alpha \in \mathcal{A}$ and $0 \leqslant i \leqslant q_{n}^{\alpha}-1$.

Lemma 1. (see [2]) Let $f \in \mathbb{B}^{1+b v}$. Then for sufficiently large $n$ there is $\lambda \in(0,1)$ such that $\left\|\xi_{n+k}\right\| \leqslant \lambda\left\|\xi_{n}\right\|$. 
The following corollary follows from Lemma 1 and states that intervals of the dynamical partition $\xi_{n}$ have exponentially small length.

Corollary 1. Let $f \in \mathbb{B}^{1+b v}$. Then for sufficiently large $n$ and $m$ with $m-n>k$ there is $\lambda \in(0,1)$ such that

$$
\left\|\xi_{n}\right\| \leqslant \lambda^{\frac{n}{k}-1} \text { and }\left\|\xi_{m}\right\| \leqslant \lambda^{\frac{m-n}{k}-1}\left\|\xi_{n}\right\| .
$$

We need the following lemma which can be easily verified.

Lemma 2. For given $a, b, c, d>0$ the following inequalities hold

$$
\min \left\{\frac{a}{b}, \frac{c}{d}\right\} \leqslant \frac{a+c}{b+d} \leqslant \max \left\{\frac{a}{b}, \frac{c}{d}\right\} .
$$

Now the well-known Doob's theorem on martingales is formulated.

Doob's Theorem ([14]). Let $\left\{\Phi_{n}\right\}$ be a sequence of random variables on a probability space $(X, F, P)$. If $\sup E\left|\Phi_{n}\right|^{p}<\infty$ for some $p>1$ and $\left\{\Phi_{n}\right\}$ is a martingale then there exists an integrable $\Phi \in \mathbb{L}_{1}(X, F)$ such that

$$
\lim _{n \rightarrow \infty} \Phi_{n}=\Phi\left(\text { a.e. P), } \Phi_{n} \rightarrow \Phi \text { in } L_{1}-\right.\text { norm }
$$

\section{Proof of the main result}

Let us consider the dynamical partition $\xi_{n}\left(f_{1}\right)$. For simplicity $L^{(n)}$ is use to denote an interval $f^{i}\left(I_{\alpha}^{(n)}\right)$ of the dynamical partition $\xi_{n}\left(f_{1}\right)$. Let us define a sequence of random variables $\left\{\Phi_{n}\right\}$ on the interval $I$ as

$$
\Phi_{n}(x)=\frac{\left|h\left(L^{(n)}\right)\right|}{\left|L^{(n)}\right|}, \quad \text { if } \quad x \in L^{(n)}
$$

Lemma 3. The sequence of piecewise functions $\left\{\Phi_{n}(x), n \geqslant 1\right\}$ generates a finite martingale with respect to the dynamical partition $\xi_{n}$.

Proof. Since conjugate map $h$ is a homeomorphism $\Phi_{n}(x)$ is a step function which takes constant values on each element $L^{(n)}$ of the partition $\xi_{n}$. Then $\Phi_{n}(x)$ is $\xi_{n}$ - measurable. Therefore, suffice it to show that

$$
E\left(\Phi_{n+1} / \xi_{n}\right)=\Phi_{n}, \text { for all } n \geqslant 1,
$$

where $E\left(\Phi_{n+1} / \xi_{n}\right)$ is a conditional expectation of the random variable $\Phi_{n+1}$ with respect to the partition $\xi_{n}$. Let us denote the indicator function of the interval $f^{i}\left(I_{\alpha}^{(n)}\right)$ by $X_{\alpha, i}^{(n)}(x)$. By definition of conditional expectation with respect to the partition we have

$$
E\left(\Phi_{n+1} / \xi_{n}\right)=\sum_{\alpha \in \mathcal{A}} \sum_{i=0}^{q_{n}^{\alpha}-1} E\left(\Phi_{n+1} / f^{i}\left(I_{\alpha}^{(n)}\right)\right) X_{\alpha, i}^{(n)}(x) .
$$

Let us recall that the partition $\xi_{n+1}$ consists of the preserving elements of $\xi_{n}$ and images of two (new) intervals for defining $R^{n+1}(f)$, that is, $\xi_{n+1}=\xi_{n+1}^{p r} \cup \xi_{n+1}^{t n}$. Spliting sum (9) in two sums corresponding to $\xi_{n+1}^{p r}$ and $\xi_{n+1}^{t n}$, we obtain

$$
E\left(\Phi_{n+1} / \xi_{n}\right)=\sum_{J_{i} \in \xi_{n+1}^{p r}} E\left(\Phi_{n+1} / J_{i}\right) X_{\alpha, i}^{(n)}(x)+\sum_{J_{i} \in \xi_{n+1}^{t n}} E\left(\Phi_{n+1} / J_{i}\right) X_{\alpha, i}^{(n)}(x) .
$$

where $J_{i}=f^{i}\left(I_{\alpha}^{(n)}\right)$. Consider the first sum that corresponds to $\xi_{n+1}^{p r}$ in (10). Then

$$
E\left(\Phi_{n+1} / J_{i}\right)=\frac{1}{\left|f^{i}\left(I_{\alpha}^{(n)}\right)\right|} \int_{f^{i}\left(I_{\alpha}^{(n)}\right)} \Phi_{n+1}(x) d x=\frac{1}{\left|f^{i}\left(I_{\alpha}^{(n)}\right)\right|} \int_{f^{i}\left(I_{\alpha}^{(n)}\right)} \Phi_{n}(x) d x .
$$


Next we consider the sum that corresponds to $\xi_{n+1}^{t n}$ in (10). Let $J_{i}:=\bigcup I_{\alpha}^{(n+1)}$, where $I_{\alpha}^{(n+1)} \in$ $\xi_{n+1}^{t n}$. Then we obtain

$$
\begin{gathered}
E\left(\Phi_{n+1} / J_{i}\right)=\frac{1}{\left|f^{i}\left(I_{\alpha}^{(n)}\right)\right|} \int_{f^{i}\left(I_{\alpha}^{(n)}\right)} \Phi_{n+1}(x) d x= \\
=\frac{1}{\left|f^{i}\left(I_{\alpha}^{(n)}\right)\right|} \sum_{I_{\alpha}^{(n+1)} \in J_{i}} \int_{I_{\alpha}^{(n+1)}} \Phi_{n+1}(x) d x=\frac{1}{\left|f^{i}\left(I_{\alpha}^{(n)}\right)\right|} \int_{f^{i}\left(I_{\alpha}^{(n)}\right)} \Phi_{n}(x) d x .
\end{gathered}
$$

This and equations in (10), (11) imply the result.

Let us introduce $\Theta_{n}(x)=\Phi_{n}(x)-\Phi_{n-1}(x), n \geqslant 1$, and put $\Phi_{0}(x):=0, x \in I$.

Lemma 4. For all $n \geqslant 1$ the following inequality holds

$$
\left|\Theta_{n}(x)\right| \leqslant \delta_{n}\left|\Phi_{n}(x)\right|, x \in I \text {, with } \delta_{n} \in l_{2} .
$$

Proof. It is clear that

$$
\left|\Theta_{n}(x)\right|=\left|\Phi_{n}(x)\right| \cdot\left|\frac{\left|\Phi_{n}(x)\right|}{\left|\Phi_{n-1}(x)\right|}-1\right| .
$$

Let $\Phi_{n}\left(L^{(n)}\right):=\Phi_{n}(x), \quad x \in L^{(n)}$. Then we have

$$
\left|L^{(n-1)}\right| \cdot \Phi_{n-1}\left(L^{(n-1)}\right)=\sum_{s=1}^{k_{n}}\left|L^{(n)}(s)\right| \cdot \Phi_{n}\left(L^{(n)}(s)\right),
$$

where $L^{(n)}(s) \subset L^{(n-1)}$. Using Lemma 2, we obtain

$$
\min \Phi_{n}\left(L^{(n)}(s)\right) \leqslant \Phi_{n-1}\left(L^{(n-1)}\right) \leqslant \max \Phi_{n}\left(L^{(n)}(s)\right) .
$$

It is clear that for any $0 \leqslant s \leqslant k_{n}$ the following is true

$$
\frac{\min \Phi_{n}\left(L^{(n)}(s)\right)}{\max \Phi_{n}\left(L^{(n)}(s)\right)} \leqslant \frac{\Phi_{n-1}\left(L^{(n-1)}\right)}{\Phi_{n}\left(L^{(n)}(s)\right)} \leqslant \frac{\max \Phi_{n}\left(L^{(n)}(s)\right)}{\min \Phi_{n}\left(L^{(n)}(s)\right)} .
$$

Since each pair of adjacent intervals of $\xi_{n}\left(f_{1}\right)$ are comparable, by assumption of Theorem 1.1 we obtain

$$
\left|\frac{\Phi_{n}\left(L^{(n)}(s+1)\right)}{\Phi_{n}\left(L^{(n)}(s)\right)}-1\right| \leqslant \delta_{n} .
$$

Hence, our map has bounded combinatorics we have

$$
\frac{\max \Phi_{n}\left(L^{(n)}(s)\right)}{\min \Phi_{n}\left(L^{(n)}(s)\right)} \leqslant\left(1+C_{2} \delta_{n}\right)^{K} \leqslant 1+C_{4} \delta_{n}
$$

One can show that lower bound holds true for the ratio $\min \Phi_{n}\left(L^{(n)}(s)\right): \max \Phi_{n}\left(L^{(n)}(s)\right)$. Then, for all $0 \leqslant s \leqslant k_{n}$, we have

$$
1-C_{4} \delta_{n} \leqslant \frac{\Phi_{n-1}\left(L^{(n-1)}\right)}{\Phi_{n}\left(L^{(n)}(s)\right)} \leqslant 1-C_{4} \delta_{n}
$$

This completes the proof of Lemma 4.

Proof of Theorem 1.1. Note that sequence $\left\{\Phi_{n}\right\}$ of random variables is a martingale with respect to $\xi_{n}\left(f_{1}\right)$ by lemma 3 . Let us show that $\Phi_{n}$ converges to $D h$ in $\mathbb{L}_{1}$-norm as $n \rightarrow \infty$. One 
can show that $\Theta_{n}(x)$ and $\Phi_{n-1}(x)$ are orthogonal, that is, $\int_{I} \Theta_{n}(x) \Phi_{n-1}(x) d x=0$. Then using Lemma 4, we have

$$
\left\|\Phi_{n}\right\|_{L_{2}}^{2} \leqslant\left\|\Phi_{n-1}\right\|_{L_{2}}^{2}+\left\|\Theta_{n}\right\|_{L_{2}}^{2} \leqslant\left(1+C_{4} \delta_{n}^{2}\right)\left\|\Phi_{n-1}\right\|_{L_{2}}^{2}
$$

Iterating the the last relation, we have $\left\|\Phi_{n}\right\|_{L_{2}}^{2} \leqslant \prod_{j=1}^{n}\left(1+C_{4} \delta_{j}^{2}\right)$. Since the series $\sum_{j=1}^{\infty} \delta_{j}^{2}$ converges then the sequence of random variables $\left\{\Phi_{n}\right\}$ is bounded in $L_{2}$ norm. Doob's theorem implies that the sequence $\left\{\Phi_{n}\right\}$ converges to some function $\Phi$ in $L_{1}$ norm.

Next we prove that the sequence of random variables $\left\{\Phi_{n}\right\}$ converges to $D h$. Let us denote the left end point of the interval $L^{(n)}$ by $\gamma_{n}$. By definition of $\Phi_{n}$ we have

$$
\left|h(x)-\int_{0}^{x} \Phi_{n}(x) d x\right| \leqslant\left|h(x)-h\left(\gamma_{n}\right)\right|+\frac{\left|h\left(L^{(n)}\right)\right|}{\left|L^{(n)}\right|}\left|x-\gamma_{n}\right| \leqslant 2\left|h\left(L^{(n)}\right)\right| .
$$

Using the last inequality, we obtain

$$
\begin{aligned}
\left|h(x)-\int_{0}^{x} \Phi(x) d x\right| \leqslant \mid h(x)- & \int_{0}^{x} \Phi_{n}(x) d x \mid+ \\
& +\int_{0}^{x}\left|\Phi(x)-\Phi_{n}(x)\right| d x \leqslant 2\left|h\left(L^{(n)}\right)\right|+\left\|\Phi_{n}-\Phi\right\|_{L_{1}} .
\end{aligned}
$$

In the limit $n \rightarrow \infty$, we obtain $h(x)=\int_{0}^{x} \Phi(x) d x$. Since, $\Phi \in L_{1}(I)$ then $h$ is an absolutely continuous function and $D h(x)=\Phi(x)$ almost everywhere on $I$. Theorem 1.1 is completely proved.

\section{References}

[1] A.Avila, M.Viana, Simplicity of Lyapunov spectra: proof of the Zorich-Kontsevich conjecture, Acta Math., 198(2007), 1-56.

[2] K.Cunha, D.Smania, Renormalization for piecewise smooth homeomorphisms on the circle, Ann. Inst. H.Poincare, Anal. Non Lin., 30(2013), no. 3, 441-462.

[3] K.Cunha, D.Smania, Rigidity for piecewise smooth homeomorphisms on the circle, Adv. in Math., 250(2014), 193-226.

[4] S.Marmi, P.Moussa, J.-C.Yoccoz, The cohomological equation for Roth type interval exchange transformations, Journal of the Amer. Math. Soc., 18(2005), 823-872.

[5] S.Marmi, P.Moussa, J.-C.Yoccoz, Linearization of generalized interval exchange maps, Ann. of Math., 176(2012), 1583-1646.

[6] M.Viana, Dynamics of interval exchange transformations and Teichmüller flows, Lecture notes of graduate courses taught at IMPA in 2005 and 2007, 2008.

[7] K.Khanin, A.Teplinsky, Renormalization horseshoe and rigidity for circle diffeomorphisms with breaks, Comm. Math. Phys., 320(2013), 347-377.

[8] K.Khanin, S.Kocić, Renormalization conjecture and rigidity theory for circle diffeomorphisms with breaks, Geom. Funct. Anal., 24(2014), no. 6, 2002-2028. 
[9] S.Kocić, Generic rigidity for circle diffeomorphisms with breaks, Comm. Math. Phys., 344(2016), no. 2, 427-445.

[10] A.Begmatov, K.Cunha, A.Dzhalilov, On the renormalizations of circle homeomorphisms with several break points, 2018.

[11] A.Begmatov, K.Cunha, On the convergence of renormalizations of piecewise smooth homeomorphisms on the circle, 2018.

[12] Y.Katznelson, D.Ornstein, The absolute continuity of the conjugation of certain diffeomorphisms of the circle, Ergod. Theor. Dyn. Sys., 9(1989), 681-690.

[13] M.Keane, Interval exchange transformations, Math. Z., 141(1975), 25-31.

[14] A.N.Shiryayev, Probability, New York, NY, USA, 1984.

\section{Достаточное условие абсолютной непрерывности сопряжений между перекладываниями интервала}

Абдумажид С. Бегматов

Национальный университет Узбекистана Университетская, 4, Олмазорский район, Ташкент, 100174 Туринский политехнический университет в Ташкенте Ниязова, 17, Ташкент, 100095 Узбекистан

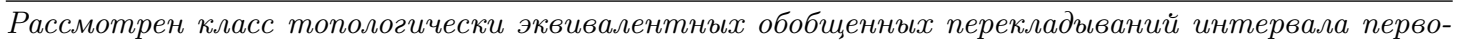
го рода с одинаковой ограниченной комбинаторикой. В статъе приведено достаточное условие абсолютной непрерьвности сопряжения между двумя отображениями из этого класса.

Ключевые слова: сопрягающий гомеоморфизм, перекладьвание интервала, индукиия Раузи-Вича, ренормализачия, динамическое разбиение, мартингал. 